\title{
Diferentes alternativas de rellenos óseos
}

\section{Different alternatives of bone grafts}

\author{
TORTOLINI P* \\ RUBIO S**
}

\begin{abstract}
Tortolini P, Rubio S. Diferentes alternativas de rellenos óseos. Av Periodon Implantol. 2012; 24, 3: 133-138.
\end{abstract}

\begin{abstract}
RESUIMEN
Los recientes avances en biotecnología, el conocimiento de los factores de crecimiento y el estudio de los distintos biomateriales han desarrollado un área que se define como ingeniería tisular entendiendo por tal a la reconstrucción de nuevos tejidos para el reemplazo y la regeneración de estructuras destruidas y perdidas.

La elección en la actividad clínica (tratamiento de problemas periodontales, cirugías o implantes) entre un material de relleno y otro exigen conocimientos de las propiedades histológicas normales de los tejidos y las características de los biomateriales.
\end{abstract}

PALABRAS CLAVE: Ingeniería tisular, biomateriales, injertos óseo.

\section{SUMMMARY}

Recent advances in biotechnology, knowledge of growth factors and the study of different biomaterials have developed an area which is defined as tissue engineering understood as the reconstruction of new tissues for replacement and regeneration of destroyed and lost infrastructure. The choice for clinical activity (treatment of periodontal problems, surgeries, or implants) between a filling material and other properties require knowledge of normal histology of tissues and the characteristics of biomaterials.

KEY WORDS: Tissue engineering, biomaterials, bone graft.

Fecha de recepción: 19 de abril de 2010.

Fecha de aceptación: 5 de mayo de 2010.

\section{INTRODUCCIÓN}

Los injertos óseos tienen una función mecánica y biológica. En la interfase injerto óseo-huésped existe una compleja relación donde múltiples factores pueden intervenir para una correcta incorporación del injerto, entre ellos se encuentran la vascularización del injerto, técnicas de conservación, factores locales, factores sistémicos y propiedades mecánicas (dependen del tipo, tamaño y forma del injerto utilizado) (1).

Como parte del tratamiento Periodontal de lesiones de Furca II y defectos 2 y 3 paredes la bibliografía describe el uso de biomateriales (2).
Así mismo para reconstrucciones dentarias a través de tratamientos implantológicos es esencial un adecuado volumen óseo para la oseointegración (3) de allí la importancia de conocer por parte del odontólogo el comportamiento del tejido óseo y los mecanismos biológicos por los cuales actúan los materiales.

Uno de los componentes destacados del sistema estomatognático es el hueso alveolar. Esta es una estructura odontodependiente, ya que se forma junto con los elementos dentarios, los sostiene mientras cumplen su función y desaparece una vez que los dientes se pierden (4). 
Dentro de los biomateriales utilizados para regeneración ósea se describen los de relleno o injerto (productos biológicos que rellenan los defectos óseos) y los materiales de aislamiento o barrera (Son biomateriales naturales o sintéticos que actuando como barrera evitan que ciertos tipos celulares invadan un espacio concreto permitiendo así la proliferación de grupos celulares específicos). Los mecanismos biológicos por los cuales actúan dichos materiales son objeto de estudios experimentales y clínicos en los últimos años.

\section{DESARROLLO}

La formación ósea inducida por biomateriales, sea cual fuere el mecanismo que la provoca, refleja principalmente una modificación en el microambiente celular (5). En general, después del establecimiento de un tejido conjuntivo inmaduro y bien vascularizado, la formación ósea continúa con un reclutamiento, proliferación y diferenciación de células osteoblásticas con secreción de colágeno, proteínas de la matriz y posterior mineralización $(6,7)$.

Específicamente el hueso alveolar se encuentra sometido a procesos continuos de remodelación regulados por factores sistémicos como la hormona paratiroidea, vitamina D3, insulina, hormona de crecimiento, glucocorticoides, proteína morfogenética ósea, factor de crecimiento transformante $B$, factor de crecimiento semejante a insulina, factor de crecimiento fibroblástico y factor de crecimiento derivado de plaquetas (8).

Cuando se colocan materiales de relleno existe una interacción entre las partículas del mismo con el ambiente que lo rodea, especialmente el tejido óseo. Este fenómeno reviste una importancia fundamental para el éxito del injerto. De allí la trascendencia del conocimiento de los componentes (Gráfico 1).

Teniendo en cuenta esta relación de matriz-células óseas y tratando de aprovechar la comunicación molecular entre ambos se han utilizado diferentes materiales que incluyen injertos óseos autólogos, materiales alogénicos, xenogénicos, sustitutos óseos, técnicas de regeneración óseas guiada y el uso de proteínas óseas morfogenéticas.

En este sentido, los diferentes materiales utilizados pueden actuar por al menos uno de estos tres mecanismos bien descritos en la bibliografía (9-14):

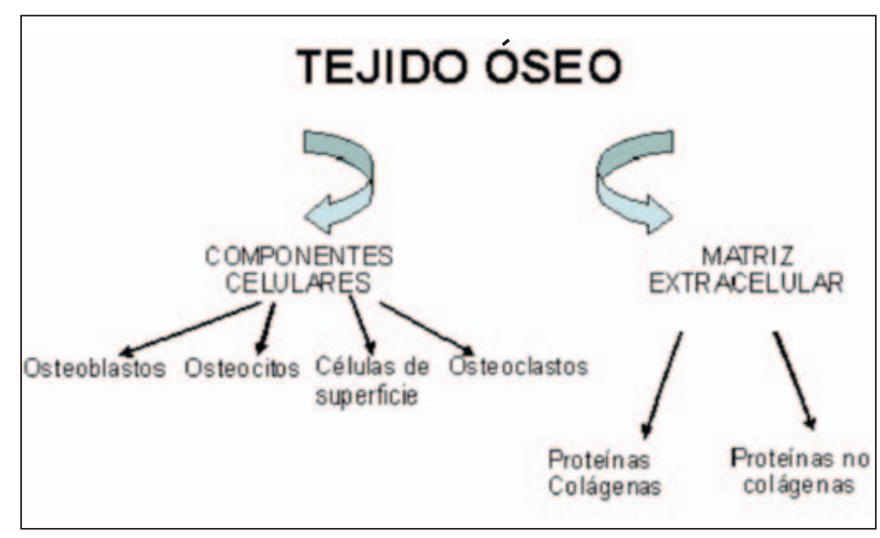

Gráfico 1: Componentes del tejido óseo. Gentileza Dr. Fontana Sebastián. Tesis Doctoral 2009.

- Osteogénesis: Síntesis de hueso nuevo a partir de células derivadas del injerto o del huésped. Requiere células capaces de generar hueso.

- Osteoinducción: Es un proceso que estimula la osteogénesis, por el que las células madres mesenquimatosas son reclutadas en la zona receptora y a su alrededor para diferenciarse en condroblastos y osteoblastos. La diferenciación y el reclutamiento son modulados por factores de crecimiento derivados de la matriz del injerto, cuya actividad es estimulada al extraer el mineral óseo.

Entre los factores de crecimiento se encuentran las proteínas morfogenéticas óseas 2,4 y 7 , factor de crecimiento derivado de las plaquetas, interleuquinas, factor de crecimiento fibroblástico, factores de crecimiento pseudoinsulínico, factores estimuladores de las colonias de granulocitos-macrófago (1). También se liberan factores angiogénicos, como el factor de crecimiento vascular derivado del endotelio y la angiogenina.

Los materiales osteoinductivos pueden hacer crecer hueso en la zona donde normalmente no se encuentra.

- Osteoconducción: Es un proceso por el cual el material provee un ambiente, estructura o material físico apropiado para la aposición de hueso nuevo. Se desencadena un crecimiento tridimensional de capilares, tejido perivascular, y células madres mesenquimatosas, desde la zona receptora del huésped hacia el injerto. Este andamiaje permite la formación de hueso nuevo mediante un patrón previsible, determinado por la biología del injerto y el entorno mecánico de la interfase huésped-injerto. 
El injerto óseo ideal debería tener estas tres propiedades además de ser biocompatible y proporcionar estabilidad biomecánica.

Se puede definir la biocompatibilidad cuando un material se considera compatible y solo provoca reacciones deseadas o tolerables en el organismo vivo (15).

Con la finalidad de lograr alguno de los procesos nombrados anteriormente, los injertos óseos han sido objetivo de estudios durante más de cuatro décadas. Entre las diferentes opciones se pueden citar $(16,17)$ :

\section{a) Injertos autólogos o autógenos}

Actúan a través de los tres mecanismos biológicos osteogénesis, osteoconducción, osteoinducción.

Es hueso obtenido del propio paciente. Puede ser de hueso esponjoso, corticales vascularizadas o corticales no vascularizadas y los distintos tipos de injertos pueden tener distintas propiedades. El mejor material de relleno es el hueso autólogo corticoesponjoso o particulado de esponjoso que puede formar hueso nuevo por mecanismo de osteogénesis, osteoconducción y tiene escasa capacidad antigénica.

Se obtienen de sitios intraorales (mentón, tuberosidad del maxilar, rama ascendente) que se usan para pequeños defectos o extraorales (cresta ilíaca, tibia o calota) cuando se requiere mayor cantidad. La elección de cada abordaje dependerá del tipo, tamaño y forma de la cavidad ósea, la experiencia clínica y preferencia del profesional. El hueso autógeno esponjoso es el que tiene mayor capacidad osteogénica y los injertos corticales son los que proporcionan mayor estabilidad (18).

Sin embargo, la obtención de autoinjertos óseos requiere un procedimiento quirúrgico en el sitio donante con el consiguiente riesgo de morbilidad postoperatoria, infección, dolor, hemorragia, debilidad muscular, lesión neurológica, entre otras. También aumenta considerablemente el tiempo quirúrgico y en algunos casos la cantidad de injerto extraído puede ser insuficiente.

También papilla de hueso, que se recoge en un filtro durante una osteoplastia/osteotomía realizada simultáneamente, se puede volver a implantar en una bolsa (15).

\section{b) Injertos homólogos, alogénicos o aloinjertos} (19-21)

Proceden de individuos de la misma especie; pero genéticamente diferentes. Se pueden clasificar según su procesamiento en:

- Aloinjertos congelados.

- Aloinjerto iofilizado (secado en frío).

- Aloinjerto iofilizado y desmineralizado.

- Hueso irradiado.

Aunque este material se promocione como osteoinductor, por los resultados obtenidos a través de estudios experimentales en tejidos extraóseos (tejido celular subcutáneo) se consideran biocompatibles y osteoconductores (5).

Las ventajas del aloinjerto incluyen su disponibilidad en cantidades importantes y diferentes formas y tamaños, no se sacrifican estructuras del huésped y no hay morbilidad del sitio donante. Las desventajas se relacionan con la calidad del tejido óseo regenerado, que no siempre es previsible. Necesitan un procesado para eliminar su capacidad antigénica $(22,23)$.

\section{c) Injertos heterólogos o xenoinjertos}

De origen natural, provienen de otra especie (animales) y contienen los minerales naturales del hueso. Se ha informado que la porosidad y la superficie de estos materiales resulta en una mejor respuesta osteogénica (24-26).

Por ejemplo, hueso bovino y derivados del coral (Ostrix, Osteogen, Bio-Oss, Interpore).

El uso de hueso mineral desproteinizado de bovino (Bio.oss, Osteohealth Suiza) ha sido estudiado y se ha comprobado que ofrece verdaderas ventajas en zonas de alta demanda estética, ya que sirve como apoyo para el tejido blando (26).

Otros estudios a largo plazo mostraron que la colocación de bio-oss en un alveolo pos exodoncia impide la contracción marginal del reborde que ocurre luego de la extracción dentaria (27).

Las propiedades del bio-oss son similares a las del hueso humano la estructura porosa del mismo ofrece espacio para las células sanguíneas y el depósito de nuevo hueso. La microestructura de la superficie del 
bio-oss soporta la adhesión de los osteoblastos que son los responsables de la formación del hueso.

\section{d) Injertos aloplásticos o sintéticos}

Provenientes de materiales fabricados sintéticamente. Se encuentran en variadas formas, tamaños y texturas Las respuestas biológicas óseas dependerán de las técnicas de fabricación, la cristalinidad, porosidad y grado de reabsorción $(28,29)$.

Pueden ser: Cerámicos: son los de uso más común, por ejemplo el fosfato de calcio sintético (hidroxiapatita y fosfato tricálcico). Polímeros: como Bioplan, HTR.Vidrio Cerámico bioactivo: compuesto de sales de calcio y fosfato, y sales de sodio y silicio (Biogass, Perioglass, Biogran) El principal mecanismo de acción de estos materiales es osteoconducción (30).

Los materiales osteoconductivos deben tener una porosidad que permita la vascularización y provea un área de adherencia a las células osteogénicas.

El tamaño del poro óptimo para que esto ocurra es entre 100 y $500 \mathrm{Mn}$ con un volumen total de poros de 75/80 además los compuestos deben ser no inmunogénicos y el hueso debe tener una capacidad alta de adherencia a los mismos.

Estos materiales han sido estudiados teniendo en cuenta su histomorfometría y biología molecular obteniendo resultados óptimos.

Un factor importante a considerar es mantener el injerto en su posición y evitar que los tejidos blandos interfieran la cicatrización ósea. Durante los primeros momentos de cicatrización del material de injerto, se produce una competición entre el tejido óseo y el blando para rellenar la cavidad y el tejido blando prolifera mas rápido tendiendo a cerrar la cavidad.

El desarrollo de las membranas de regeneración ósea guiada ha demostrado su utilidad para asistir y ayudar en los injertos óseos (31).

Dentro de los materiales de barrera, encontramos las membranas para osteopromoción (32).

La osteopromoción es el sellado por medios físicos de un sitio anatómico, para impedir que otros tejidos invadan el coagulo óseo e interfieran con la regeneración ósea. Este es el mecanismo por el cual actúan las membranas de regeneración tisular guiada. En la actualidad existen dos grupos de membranas para regeneración.

- Reabsorbibles. Estas membranas presentan capacidad de ser reabsorbidas por el organismo. El periodo de reabsorción depende del material que las constituye, esto es un punto crítico dado que al no ser necesaria su remoción, su función depende del tiempo que permanezcan en el organismo.

Se clasifican de acuerdo a su composición en:

- Colágeno: Obtenido de tendón bovino purificado (colágeno tipo 1), ej.: Biomed (Zimer-USA) se reabsorbe aproximadamente a las 6 o 7 semanas.

- PLA-PGA: (ácido poliláctico-ácido poli glicólico) son mas rígidas y su tiempo de reabsorción es de 6 a 8 semanas, ej.: Resolut (Goretex USA)

- Polímero líquido sintético.

- Poliglactina.

- Sulfato de calcio.

- No reabsorbibles. Son membranas constituidas por teflón (politetrafluoruro de etileno PTFE) De acuerdo al tratamiento del material pueden ser expandidas o no. Estas membranas poseen la desventaja de requerir una segunda cirugía para su remoción, que se puede acelerar en caso de exposición o infección.

El periodo ideal de mantenimiento de la membrana debe ser de 6 meses, pero se puede modificar según el caso clínico en particular.

\section{CONCLUSIÓN}

Dada la demanda cada vez mayor de los injertos óseos han surgido alternativas al injerto óseo autólogo, siendo este el ideal por su gran capacidad osteogénica.

Si bien los mas utilizados son los aloinjertos y xenoinjertos; se encuentran en desarrollo alternativas sintéticas conocidas como sustitutos óseos. De manera ideal éstos deben ser biocompatibles, biodegradables, osteoconductivos y osteoinductivos con una estructura similar al hueso de bajo costo y fácil uso.

Todo material de implantación debe desencadenar una reacción lo más fisiológicamente posible con los tejidos que lo rodean. Es fundamental conocer los procesos biológicos normales que se desencadenan en la 
regeneración y las características físicas, mecánicas y biológicas propias de cada material.

De todo lo expuesto y por la amplia oferta de biomateriales que existen en el mercado es fundamental analizar los trabajos clínicos y experimentales que avalen científicamente el comportamiento y mecanismo de acción de estos materiales destacando la importancia del concepto de biocompatibilidad.

\section{BIBLIOGRAFÍA}

1. Barón Zárate-Kalfópulos, Reyes Alejandro-Sánchez. Injertos óseos en cirugía ortopédica. Cir Ciruj 2006;74: 217-22.

2. Deliberador T, Nagata M, Furlaneto F, Melo Oo, Sundefeld M, Fucini S. Autogenous Bone Graft With or whitou a Calcium Sultate Barrier in treatment of class II Furcation Defects: A Histologic and Histometric Study in Dogs. J Periodontology May 2006;77(5):780-8.

3. Molly L, Vandromme $\mathrm{H}$, Quirynen $\mathrm{M}$ et al. Bone formation following implantation of bone biomaterials into extraction sites. J Periodontol. 2008;79:1108-15.

4. Gómez de Ferraris ME, Campos Muñoz A. Periodoncio de Inserción: Cemento, Ligamento Periodontal y Hueso Alveolar. En: Histología y embriología bucodental. Ed Médica Panamericana. Madrid, 2002: $2^{\mathrm{a}}$ ed., pp 36883.

5. Fontana S. Respuesta biológica de los rellenos óseos. Estudio Experimental. (tésis doctoral): Facultad de Odontología, Universidad de Cordoba.2009 pp 20-8.

6. Ten Cate AR. Periodonto. En Histología Oral. Desarrollo, estructura y función.. Ed. Médica panamericana. Buenos Aires, 1986, $2^{a}$ ed., pp 20-30.

7. Cooper L. Biologic Determinants of bone formation for oseointegration: Clues for future clinical improvements. The Journal of Prosthetic Dentistry. 1998; 80: 439-449.

8. Gartner LP, Hiatt JM. Cartílago y Hueso. En: Texto Atlas de Histología. Ed Mc. Graw-Hill. Madrid, 2007, $3^{\text {a ed., }}$ pp 131-56.

9. Ripamonti U, Ramoshebi L, Maatsaba T, et al. Bone induction by BMPs/OPs and related family members in primates. The Journal of Bone and Joint Surgery 2001. Vol. 83-A, Supplement 1 part 2: 116-27.
10. Wikesjo U, Sorensen R, Wozney J. Augmentation of alveolar bone and dental implant osseointegration: Clinical implications of Studies with RH BMP-2. The Journal of Bone and Joint Surgery $2001 ; 83-A$, Supplement 1 part 2: 116-27.

11. Goldring SR, Roelke M, Glowacki J. Multinucleated cells elicited in response to implants of devitalized particles possess receptors for calcitonin. Bone Miner Res. 1988; 3:117-20

12. Marx RE, Carlson ER, Eichstaedt RM, et. al. Platelet-rich plasma. Growth factor enhancement for bone graft. Oral Surg Oral Med Oral Patho Oral Radiol Endod 1998;85: 638-46.

13. Anitua E. Plasma rich in growth factors: Preliminary results of use in the preparation of future sites for implants. Int J Oral Maxillofac Implants 1999;14:52935.

14. Lang NP, ecker W, Karring T. Formación del hueso alverolar. En: Lindhe J, eds. Periolontología clínica e implantología odontológica. Ed. Médica Panamericana Madrid, $20003^{a}$ ed. pp 916-48.

15. Klaus H Rateistschak Edith M Rateitschok-Plus Herbert F Wolf Editorial Savat. Atlas de periodoncia pp 215-25.

16. Fontana S, Olmedo D, Crosa ME, y col. Effect of Platelet Rich Plasma on the Peri-implant Bone Response. An Experimental Study. Implant Dent. 2004;13:73-8.

17. Glowacki J, Mulliken JB. Demnineralized bone implants. Clin Plast Surg. 1985;12:233-41.

18. P Infante-Cossio, Gutiérrez-Pérez, Torres-Lagares, García A, García Perla, Gonzales-Padilla. Relleno de cavidades óseas en cirugía maxilofacial con materiales autólogos. Rev. Esp Cir. Oral y Maxilof 2007;29:7-18.

19. Molly L, Vandromme H, Quirynen M Schepers E, Adams JL, Van Steenbergh Bone Formation following implantation of bone biomaterials into extraction sites J Periodontol 2008;Jun 79 (6): 1 108-15.

20. S. Ochandiano Caycoya Relleno de cavidades óseas en cirugía maxilofacial con materiales aloplásticos. Rev. Española Cirugía Oral y Maxilofacial 2007;29(1):21-32.

21. Schawartz Z, Mellonig JT, carnes DL y colab. Ability of comercial demineralized freeze-dried bone allograft to induce new formation. J Periodontol 2006;67:918-26. 
22. Peetz M. Characterization of xenogenic bone material (Appendix) to: Boyne PJ, ed. Osseous Reconstruction of the maxilla and the mandible:Surgical techniques using titanium mesh and bone mineral. Quintessence Books 1997;87-100.

23. Becker W, Becker BE, Caffesse R. A comparison of demineralized freeze-dried bone and autologous bone to induce bone formation in human extraction sockets. J Periodontol 1994;65:1128-33.

24. Eid K, Zelicof S, Perona BP, Sledge CB, Glowacki J. Tissue reactions to particles of bone substitute materials in intraosseous and heterotopic sites inn rats: discrimination of osteoinduction, osteocompatibility and inflammation. J Orthop Res. 2001;19:962-9.

25. Oeetz M Caracterization of xenogenic bone material to Boyne PJ, ed Osseous Reconstruction of the maxilla and the mandible Durgical techniques using titanium mesh and bone mineral, 1997;87-100.
30. Bucholz RW. Non allografts osteoconductive bone grafts substitutes. Clin orthop 2002;395:44-52.

31. Spagnoli DB Mazzonetto R, Marcheta JM Clinical Procedures currently using bone grafting with guided tissue regeneration techniques. Oral Maxillofac. Surg Clin North am 2001;13:423-36.

32. Fernández Boderau E, Bessone L, Tortolini P. Torassa D. Regeneración ósea Guiada. Caso clínico con tratamiento multidisciplinario. Claves de Odontología 2009;64: 34-42.

\section{CORRESPONDENCIA}

Patricia Tortolini

Ituzaingo 1035

(5000) Cordoba, Argentina.

Correo electrónico: patriciatortolini@live.com.ar 